\title{
Caracterização de uma Rede Sem-fio de Baixa Potência e Longo Alcance para Internet das Coisas
}

\author{
Fernando M. Ortiz ${ }^{1}$, Pedro Cruz ${ }^{1}$, \\ Rodrigo de S. Couto ${ }^{1,2}$ e Luís Henrique M. K. Costa ${ }^{1 *}$ \\ ${ }^{1}$ Universidade Federal do Rio de Janeiro - PEE/COPPE/GTA \\ ${ }^{2}$ Universidade do Estado do Rio de Janeiro - PEL/DETEL/FEN \\ \{fmolano, cruz, luish\}@gta.ufrj.br, rodrigo.couto@uerj.br
}

\begin{abstract}
LoRa technology, which is increasingly employed in the Internet of Things, implements low-power and long-range networks. Depending on these characteristics, LoRa may or may not be suitable for certain applications. Thus, it is important to understand how power and range depend on network parameters and the scenario. This work analyses the communication between LoRa nodes and their performance according to the distance between them and the spreading factor. The latter is a parameter of the LoRa modulation that reduces the rate of loss with consequent reduction of the transmission rate. For example, the results show that increasing this factor from 7 to 11 reduces the loss rate from $50 \%$ to $13 \%$ for transmissions at a distance of $2 \mathrm{~km}$.
\end{abstract}

Resumo. A tecnologia LoRa, que é cada vez mais empregada em Internet das Coisas, implementa redes de baixa potência e longo alcance. Dependendo dessas características, a LoRa pode ser adequada ou não para certas aplicações. Assim, é importante entender como potência e alcance dependem de parâmetros da rede e do cenário utilizado. Este trabalho analisa a comunicação entre nós LoRa e seu desempenho de acordo com a distância entre eles e o fator de espalhamento. Esse último é um parâmetro da modulação LoRa que reduz a taxa de perda com consequente redução da taxa de transmissão. Por exemplo, os resultados mostram que o aumento desse fator de 7 para 11 reduz a taxa de perda de $50 \%$ para $13 \%$, para transmissões a uma distância de $2 \mathrm{~km}$.

\section{Introdução}

Apoiados no paradigma da Internet das Coisas (Internet of Things - IoT), objetos com capacidade de sensoriamento, processamento e comunicação podem realizar tarefas e se comunicar com outros objetos ou dispositivos. Dependendo da aplicação, a comunicação pode ser dispositivo a dispositivo (Device-to-Device - D2D) ou pode tornar tais objetos acessíveis através da Internet [Atzori et al., 2010]. Esses objetos são denominados objetos inteligentes. No contexto de Cidades Inteligentes, esse paradigma tem impulsionado a experimentação e análise de infraestruturas urbanas que permitam a inovação em sistemas de mobilidade, energia e saúde, entre outros, para servir a população [Schaffers et al., 2011].

\footnotetext{
${ }^{*}$ Este trabalho foi parcialmente financiado pela CAPES, CNPq, FAPERJ e pelos processos $n^{\circ}$ 15/244948 e n ${ }^{\circ}$ 15/24490-2, da Fundação de Amparo à Pesquisa do Estado de São Paulo (FAPESP).
} 
Os objetos inteligentes têm como característica recorrente a limitação em termos de processamento e energia, tornando a comunicação sem-fio um importante desafio. As redes LoRaWAN têm como objetivo atender a esse desafio, sendo um tipo de redes de longo alcance e baixa potência, ou LPWAN (Low Power Wide Area Network). Redes LoRaWAN são implementadas utilizando a tecnologia LoRa, uma tecnologia de caráter proprietário. Outra solução similar também proprietária são as redes SigFox. Este artigo foca na tecnologia LoRa.

A técnica de modulação do sinal de rádio utilizada pelo LoRa é o espalhamento espectral [Semtech Corporation, 2015]. Nesta técnica, o sinal original é espalhado no campo da frequência, aumentando a robustez do sinal a interferências externas. Na especificação do LoRa, o espalhamento espectral está relacionado a um parâmetro denominado fator de espalhamento (Spreading Factor - SF). O LoRa pode ser configurado com seis valores diferentes para o SF (SF7, SF8, SF9, SF10, SF11 e SF12) [LoRa-Alliance, 2015b]. Para fator de espalhamento existe um compromisso entre a robustez da modulação a interferências e a taxa de transmissão de bits. Por um lado, o aumento da taxa de transmissão aumenta a vazão da comunicação. Por outro, a perda de robustez a interferências pode induzir a perdas de mensagens, reduzindo a vazão.

Neste trabalho, é realizada uma avaliação da capacidade de comunicação da tecnologia LoRa. Esta análise é realizada em função do parâmetro de espalhamento espectral, dado que esta é uma particularidade da tecnologia: a escolha da modulação configurável por software. Como parte deste trabalho, são desenvolvidos protótipos de nós de comunicação LoRa. A comunicação entre eles é testada para diferentes fatores de espalhamento, a diferentes distâncias de comunicação. A taxa de perda de mensagens e a vazão da comunicação são medidas, a fim de avaliar a eficácia de cada fator de espalhamento, para cada distância. Os resultados obtidos mostram que o aumento desse fator de 7 para 11 reduz a taxa de perda de $50 \%$ para $13 \%$, para transmissões a uma distância de $2 \mathrm{~km}$. Além disso, também é avaliada a qualidade do enlace em termos da relação sinalruído (Signal-to-Noise Ratio - SNR), verificando que o enlace está dentro dos parâmetros de operação da tecnologia.

Diferentes soluções têm envolvido o uso da tecnologia LoRa com o objetivo de cobrir grandes áreas geográficas, em diferentes ambientes. Assim, faz-se necessário avaliar características fundamentais da tecnologia, com o objetivo de aproveitar a máxima capacidade e eficiência dos dispositivos que implementam o LoRa. Alguns trabalhos da literatura têm avaliado o comportamento do LoRa em presença de velocidade e efeito Doppler [Petäjäjärvi et al., 2017], em ambientes indoor [Neumann et al., 2016] e outdoor [Petäjäjärvi et al., 2015], geolocalização [Baharudin e Yan, 2016], todos focados em avaliar o desempenho da LoRa em diferentes ambientes. Assim, o presente artigo complementa a literatura ao realizar uma avaliação do fator de espalhamento, parâmetro de configuração importante da camada física do protocolo, e investigar como a variação desse parâmetro influencia o desempenho da rede, de acordo com as condições de potência e distância entre os nós. Entre outras métricas, verifica-se o impacto da escolha do fator de espalhamento na variação da vazão obtida na rede e da perda de pacotes.

Este trabalho está organizado da seguinte forma. A Seção 2 descreve a tecnologia LoRa, mostrando a camada física (LoRa PHY) e o protocolo que define a arquitetura de comunicação e de acesso ao meio do LoRa, o LoRaWAN. A Seção 3 discute os trabalhos 
relacionados. Já a Seção 4 apresenta a metodologia de medidas utilizada neste trabalho, assim como o protótipo de hardware, baseado no Arduino, implementado para os experimentos. A Seção 5 mostra os experimentos e resultados obtidos. Por fim, a Seção 6 conclui o trabalho e apresenta desafios futuros.

\section{Tecnologias LoRa e LoRaWAN}

LoRa (Long Range) ${ }^{1}$ é uma especificação proprietária para redes de grande alcance e de baixa potência, focada em "coisas"(things), isto é, dispositivos presentes no cotidiano dos usuários e que seguem o paradigma IoT. O LoRa define uma camada física (PHY) que procura atender aos requisitos de baixo consumo de energia dos objetos inteligentes e é utilizada para implementar o protocolo LoRaWAN.

O LoRaWAN define a arquitetura do sistema e parâmetros de comunicação e de acesso ao meio (Medium Access Control - MAC) que utiliza a camada física LoRa. O LoRaWAN define taxas de velocidade de dados, suporte à comunicação bidirecional e oferta de serviços de mobilidade e localização dos nós da rede. Diferente do LoRa PHY, a especificação do protocolo LoRaWAN é aberta e divulgada ao público desde 2015.

A rede LoRaWAN é composta por três tipos de dispositivos: terminais, gateways e servidores. Os dispositivos terminais são objetos inteligentes, tipicamente, com restrições energéticas. Podem ser sensores, atuadores ou outros tipos de dispositivos inteligentes. Os gateways são dispositivos com restrições mais leves em termos de energia e processamento, que servem de ligação entre o LoRaWAN e outras redes. Os servidores são dispositivos que recebem e analisam as informações enviadas pelos dispositivos terminais ou que comandam os dispositivos terminais [Bankov et al., 2016, Raza et al., 2017, Bor et al., 2016]. Ademais, o LoRaWAN possui uma topologia em estrela de estrelas. A Figura 1 ilustra a topologia de uma rede LoRaWAN. A comunicação em tecnologia LoRa se dá entre os dispositivos terminais e os gateways.

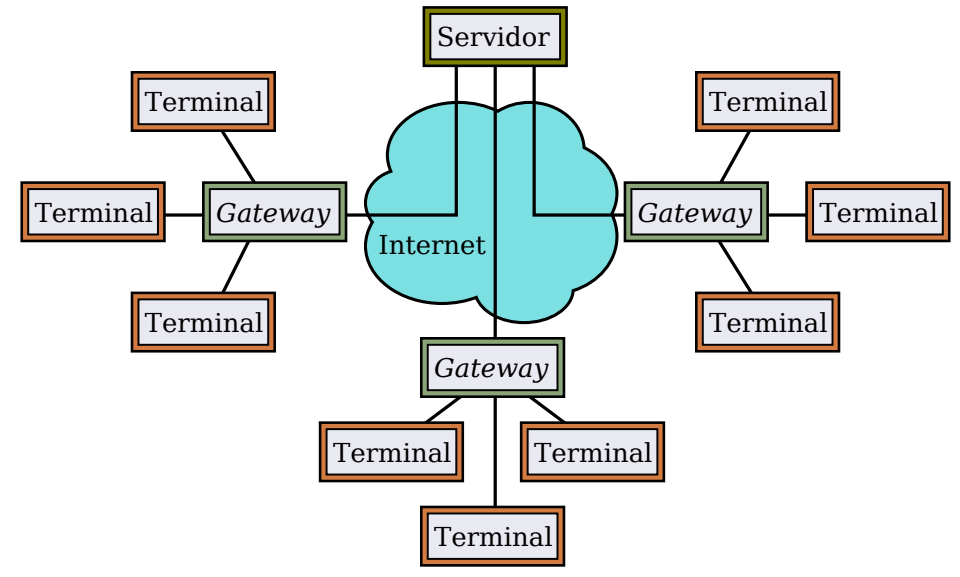

Figura 1. Exemplo de topologia para uma rede LoRaWAN.

A especificação das redes LoRaWAN define três classes de dispositivos terminais em sua arquitetura para atingir diferentes tipos de serviço: classe A, classe B, e classe C [Centenaro et al., 2016]. Nos dispositivos classe A o nó terminal pode enviar mensagens para o nó gateway a qualquer momento. Entretanto, o dispositivo terminal só fica

\footnotetext{
${ }^{1}$ https://www.lora-alliance.org
} 
disponível para recepção durante janelas de tempo denominadas janelas de recepção. Durante essas janelas, o gateway pode enviar mensagens para o dispositivo terminal. Um dispositivo classe A inicia a comunicação com o gateway e, após a transmissão, inicia uma janela de recepção, aguarda um determinado intervalo de tempo e inicia uma segunda janela de recepção. Uma nova janela de recepção apenas será aberta após uma nova transmissão do mesmo dispositivo classe A. Esse modo de operação deve ser implementado por todos os dispositivos LoRa. Nos dispositivos classe B, o processo é semelhante ao da classe A. O dispositivo classe B também abre duas janelas de recepção após realizar uma transmissão. Porém, adicionalmente, os dispositivos classe B abrem janelas de recepção com tempos agendados, configurados através de mensagens de beacon emitidas pelo gateway. Os beacons também são responsáveis pelo sincronismo entre os dispositivos terminais e os dispositivos gateway. Nos dispositivos classe C, os dispositivos terminais estão sempre disponíveis para a recepção de mensagens [Bor et al., 2016].

Uma vez que é necessário consumir energia para realizar a recepção de mensagens, as classes de operação se diferenciam não só pelo caráter da bidirecionalidade da comunicação, mas também pelo consumo de energia. A operação em modo classe A, por exemplo, pode servir a dispositivos que sejam sensores e cujo tráfego de dados seja majoritariamente a entrega de dados coletados ao gateway, com o menor custo de energia. Um exemplo de aplicação para a operação em classe B é em dispositivos que sejam sensores e atuadores que, a intervalos regulares, recebam ordens de atuação, com um custo energético intermediário. O modo de operação em classe $\mathrm{C}$, por outro lado, serve para sensores e atuadores que possam receber ordens de atuação a qualquer momento, a um custo maior de energia.

Também é definido um ciclo de trabalho (duty cycle) para os dispositivos, que é específico para cada região, uma vez que os órgãos de regulação de cada país possuem regras específicas. Este trabalho não avalia o ciclo de trabalho e a relação entre o ciclo de trabalho e o desempenho de uma rede LoRaWAN.

A camada física (PHY) da tecnologia LoRa modula sinais em sub-bandas de rádio da faixa de frequências não licenciadas ISM (Industrial, Scientific and Medical) na ordem dos MHz (União Europeia: $868 \mathrm{MHz}$ e $433 \mathrm{MHz}$, Estados Unidos da América: $915 \mathrm{MHz}$ e $433 \mathrm{MHz}$ ) [Bor et al., 2016, Centenaro et al., 2016]. Para o Brasil, de acordo com a Agência Nacional de Telecomunicações (Anatel), a faixa de frequência regulamentada para ISM é entre $902 \mathrm{MHz}$ e $928 \mathrm{MHz}$. O LoRa PHY usa correção antecipada de erros (Forward Error Correction - FEC) e uma técnica de modulação de espalhamento espectral proprietária, uma variante do espalhamento espectral por chirp (Chirp Spread Spectrum - CSS), que usa um pulso para fazer varredura das frequências, a fim de expandir o sinal espectral [Bor et al., 2016, LoRa-Alliance, 2015a].

\subsection{Parâmetros de Configuração do LoRa}

$\mathrm{O}$ consumo de energia, a faixa de transmissão e a resistência à interferência do ruído podem ser determinados a partir de quatro parâmetros de configuração do da camada física do LoRa: a frequência da portadora, que define a frequência central para a banda de transmissão; a largura de banda, que define o tamanho da faixa de frequências utilizada; a taxa de código (Code Rate - CR), que define a taxa de FEC e o fator de espalhamento (Spreading Factor - SF), que define o espalhamento espectral [Semtech Corporation, 2013, Bor et al., 2016]. 
A frequência da portadora é definida de acordo a região de operação dos equipamentos. Assim sendo, esse parâmetro não é, em geral, ajustável de acordo com aplicações. A largura de banda (BW), por sua vez, possui três larguras programáveis: $125 \mathrm{kHz}$, $250 \mathrm{kHz}$ e $500 \mathrm{kHz}$ nas especificações gerais do LoRa. A largura de banda com maior capacidade de transmissão de dados é a de $500 \mathrm{kHz}$. Assim sendo, este trabalho considera apenas a largura de banda de $500 \mathrm{kHz}$.

A taxa de código (CR) é relacionada com a técnica de FEC. A CR define quantos bits são utilizados para causar redundância na mensagem, a fim de realizar a recuperação de erros. A CR define a taxa de codificação $T_{c}$ [Semtech Corporation, 2015] como:

$$
T_{c}=\frac{4}{4+C R}, \quad \operatorname{com} C R \in\{1,2,3,4\} .
$$

A especificação LoRa define seis valores diferentes para o parâmetro de fator de espalhamento: SF7, SF8, SF9, SF10, SF11 e SF12 [Semtech Corporation, 2015]. Também é definida uma taxa de transmissão $R_{b}$ teórica, definida em função do fator de espalhamento como:

$$
R_{b}=S F \times \frac{T_{c} \times B W}{2^{S F}}, \quad \operatorname{com} S F \in\{7,8,9,10,11,12\} .
$$

A Tabela 1 exibe os valores teóricos de taxa de transmissão de diferentes fatores de espalhamento, para uma CR igual a 4/5 e uma largura de banda de $500 \mathrm{kHz}$. Esses valores teóricos são utilizados como referência para a análise de desempenho contida neste trabalho. A Seção 4 compara esses valores com valores obtidos experimentalmente, a fim de estabelecer bases para as aplicações que utilizam LoRa e LoRaWAN como tecnologias de comunicação.

Tabela 1. Valores teóricos de taxa de transmissão em função do SF.

\begin{tabular}{lc}
\hline Fator de espalhamento & Taxa de transmissão teórica (bits/s) \\
\hline SF7 & 21875 \\
SF8 & 12500 \\
SF9 & 7031 \\
SF10 & 3906 \\
SF11 & 2148 \\
SF12 & 1172 \\
\hline
\end{tabular}

\section{Trabalhos Relacionados}

Os autores de [Petäjäjärvi et al., 2017] realizam uma análise teórica e experimental do LoRa em redes LPWAN. São avaliadas métricas de desempenho utilizando um dispositivo final móvel para se observar a influência do efeito Doppler, caracterizar a taxa de entrega do LoRaWAN baseado em um modelo teórico e, por fim, realizar uma avaliação de métricas de desempenho em experimentos práticos. Petajajarvi et al. consideram o modelo de efeito Doppler definido como a variação da frequência de uma fonte deslocando-se e emitindo com respeito a um receptor estático. Em razão da técnica de modulação do LoRa, o espalhamento espectral por chirp, se a taxa de chirp é alta, isto faz com que as mudanças de tempo geradas pelas variações de frequência do efeito Doppler 
não sejam significativas, fazendo com que o desempenho do LoRa pouco se deteriore em presença do efeito Doppler. Porém, uma taxa de chirp baixa produzirá uma mudança de tempo significativa e, portanto, uma taxa de recepção baixa. Em uma segunda fase, é caracterizada a taxa de entrega de um dispositivo terminal (End Device - ED). Para tanto, é avaliado o tempo de um quadro LoRaWAN para a modulação LoRa e a modulação GFSK (Gaussian Frequency Shift Keying), visto que o LoRa está visado para oferecer uma sensibilidade de recepção maior sobre o GFSK.

Partindo dessa avaliação, o tamanho da carga útil máxima dependerá do modo de transmissão configurado no ED. Também é avaliada a taxa de entrega em presença dos regulamentos de frequência para bandas não licenciadas ISM, e por último, Petajajarvi et al. avaliam a escalabilidade e capacidade da rede. Foram feitos experimentos práticos em três cenários: (i) um cenário com um nó deslocando-se em um eixo horizontal para gerar variações de velocidade angular, (ii) um nó em um veículo comunicando-se com um receptor estático, e (iii) um nó em um ambiente veicular e um nó em um transporte aquático para medir a cobertura exterior. Este trabalho busca relacionar o fator de espalhamento com as mudanças de potência, sem focar na mobilidade constante da unidade transmissora.

Em [Baharudin e Yan, 2016], os autores implementam um protótipo com Dragino LoRa nos dispositivos terminais, e um dock (semelhante a um gateway), porém, sem as restrições de ciclo de trabalho. A potência do sinal recebido (Received Signal Strength Indication - RSSI) dos dispositivos terminais é avaliada de acordo com a variação da distância para o dock. Os dados enviados entre os nós correspondem a informações de GPS (latitude, longitude, velocidade, curso e marca de tempo (timestamp)). Os autores demonstram como a coexistência de múltiplos sensores pode degradar a qualidade do sinal de recepção, devido à interferência de redes de sensores vizinhas. No entanto, os resultados do RSSI do sinal da rede de sensores permitiram concluir que, devido à sensibilidade de recepção do LoRa, esta é uma tecnologia sem-fio apropriada para grandes distâncias. Este trabalho identifica o comportamento da potência do sinal recebido e a relação direta com a distância que o LoRa pode atingir, mas também avalia as implicações do fator de espalhamento no desempenho do enlace em relação a capacidade máxima, vazão e qualidade do sinal.

Os autores de [Centenaro et al., 2016] fazem uma comparação de diferentes tecnologias de redes de baixa potência e longo alcance (Low Power Wide Area Network LPWAN) e é realizada uma descrição específica da tecnologia LoRa. São executados dois experimentos: primeiramente, é implementada uma rede privada LoRa para controlar as variáveis de temperatura e umidade de um edifício de 19 andares. A seguir, é realizada uma análise de cobertura e alcance. Para isso, Centenaro et al. implementam uma rede LoRa em Padova, Itália, com uma área de $100 \mathrm{~km}^{2}$. Baseado no alcance teórico de um nó (em torno de $2 \mathrm{~km}$ ), foi assumida uma cobertura nominal de $1,2 \mathrm{~km}$. Conclui-se que com 30 gateways seria possível cobrir até 7.000 habitantes por gateway.

Outros trabalhos apresentam experimentos com a tecnologia LoRa em diferentes áreas de aplicação. Em [Toldov et al., 2016], os autores utilizam a tecnologia LoRa para o rastreamento de animais selvagens. Já os autores de [Kim et al., 2016] avaliam o desempenho do LoRa com respeito à distância, comparando a tecnologia com o IEEE 802.11, obtendo maior sensibilidade em recepção, porém mais baixa taxa de dados, como espe- 
rado. Por outro lado, [Neumann et al., 2016] implementam uma rede protótipo LoRa com um gateway, um dispositivo terminal, e um servidor de rede LoRa, para avaliar o desempenho em uma edificação. O nó é deslocado por diferentes pontos e diferentes andares dentro da edificação. São avaliados o rendimento, a sensibilidade de recepção, a relação sinal-ruído, a perda de pacotes, o consumo energético e o atraso de transmissão.

O presente trabalho faz uma avaliação da tecnologia LoRa, analisando as métricas de desempenho de taxa de perda, a SNR, a potência do sinal e a vazão da rede em função dos diferentes fatores de espalhamento e diferentes distâncias entre os nós. Tais experimentos têm o intuito de compreender como as variações de distância estabelecem condições diferentes para o comportamento de uma rede LoRa e LoRaWAN. Além disso, preenchem uma lacuna da literatura, pois avaliam o desempenho do LoRa em função de diferentes configurações de modulação na camada física.

\section{Metodologia de Avaliação da Tecnologia LoRa}

Os experimentos realizados para a avaliação da tecnologia LoRa consistem de medições práticas de taxa de perda, potência do sinal e relação sinal-ruído (SNR), em função de diferentes configurações do fator de espalhamento utilizado na modulação. $O$ objetivo é estabelecer parâmetros de funcionamento dos dispositivos usados e verificar a viabilidade de implementação para diferentes cenários da Internet das Coisas.

\subsection{Protótipo Experimental Desenvolvido}

A fim de realizar a avaliação desejada, um protótipo foi implementado com duas unidades de comunicação LoRa. Uma delas é configurada para enviar mensagens e é denominada unidade transmissora. A arquitetura da unidade transmissora pode ser vista na Figura 2(a). A unidade transmissora possui um controlador que gerencia as operações de sensoriamento, localização e comunicação. O sensoriamento é executado pelo sensor de temperatura e umidade, a localização é provida pelo receptor GNSS (Global Navigation Satellite System) e a comunicação é realizada pela interface sem-fio LoRa.

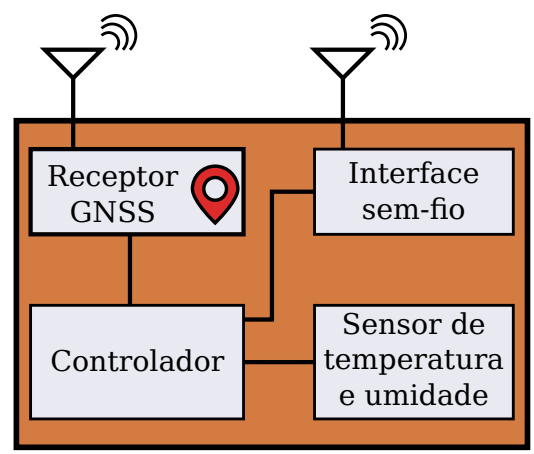

(a) Arquitetura da unidade transmis- (b) Arquitetura da unidade receptora. sora.

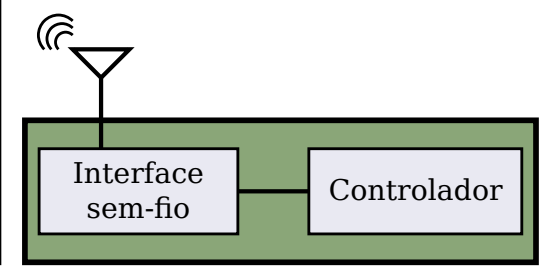

Figura 2. Arquitetura do protótipo desenvolvido.

A outra unidade é configurada apenas para receber mensagens e é denominada unidade receptora. A arquitetura da unidade receptora é ilustrada na Figura 2(b). Esta é mais simples, composta basicamente de um controlador e uma interface sem-fio LoRa. 
A Tabela 2 fornece a especificação dos componentes da unidade transmissora implementada para os experimentos. O controlador da unidade transmissora consiste em um Arduino Uno. O controlador está conectado a um módulo de GPS e a um módulo LoRaWAN da Dragino, ambos no formato de escudo (shield). O módulo LoRaWAN é baseado na placa RF96 da Semtech, que opera na frequência de $915 \mathrm{MHz}$. É utilizada uma antena de $7 \mathrm{dBi}$, do fabricante D-Link, para a transmissão do sinal LoRa. A Figura 3(a) mostra o protótipo físico da unidade transmissora.

Tabela 2. Componentes utilizados na unidade transmissora.

\begin{tabular}{l|l|l}
\hline Módulo & Equipamento & Fabricante \\
\hline Controlador & Arduino Uno R3 & Arduino \\
Receptor GNSS & U-blox NEO-6M & DuinoPeak \\
Interface sem-fio & LoRa Shield RF96 & Dragino \\
Antena LoRa & Antena 7dBi & D-Link \\
Sensores & Sensor de Umidade DHT22 & Aosong \\
& Sensor de Temperatura DHT22 & Aosong \\
\hline
\end{tabular}

A unidade receptora, cujos equipamentos estão listados na Tabela 3, consiste em um Arduino Yún conectado a um módulo LoRaWAN da Dragino, idêntico ao módulo utilizado na unidade transmissora. Assim como na unidade transmissora, é utilizada uma antena de $7 \mathrm{dBi}$ de fabricação da D-Link, porém, para a recepção do sinal LoRa. A Figura 3(b) mostra o protótipo físico da unidade receptora.

Tabela 3. Componentes utilizados na unidade receptora.

\begin{tabular}{l|l|l}
\hline Módulo & Equipamento & Fabricante \\
\hline Controlador & Arduino Yún & Arduino \\
Interface sem-fio & LoRa Shield RF96 & Dragino \\
Antena LoRa & Antena 7 dBi & D-Link \\
\hline
\end{tabular}

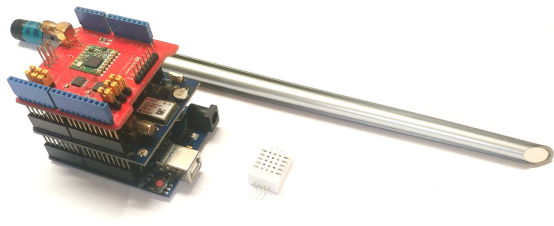

(a) Unidade transmissora.

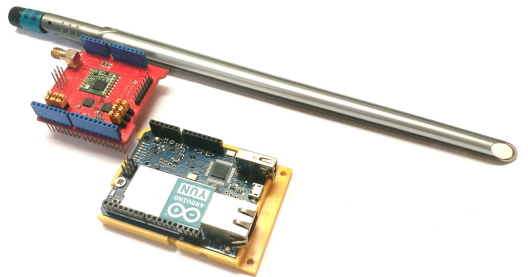

(b) Unidade receptora

Figura 3. Protótipos do experimento.

O módulo LoRa utilizado permite a comunicação com o Arduino por meio do protocolo SPI (Serial Peripheral Interface Bus). O módulo de GPS utiliza comunicação serial com o controlador Arduino UNO. As unidades transmissora e receptora são programadas como dispositivos classe A, utilizando a biblioteca RadioHead ${ }^{2}$. A potência de transmissão foi estabelecida em $14 \mathrm{dBm}$, a largura de banda é configurada para $500 \mathrm{kHz}$ e o CR utilizado é $4 / 5$.

\footnotetext{
${ }^{2}$ https://github.com/PaulStoffregen/RadioHead.
} 
O controlador da unidade transmissora é programado de forma que, para cada valor de SF programado, sejam transmitidos pacotes, numerados de 0 a 50. Os pacotes são preenchidos com a numeração, as coordenadas de posição indicadas pelo GPS, assim como com a temperatura e a umidade medidas pelo sensor. O espaço restante em cada pacote é preenchido com caracteres aleatórios até que o mesmo esteja cheio. Os valores de SF programados são SF7, SF8, SF9, SF10 e SF11. É importante notar que os tamanhos de pacote variam de acordo com o valor utilizado para o SF. A Tabela 4 mostra os tamanhos de quadros usados para o experimento; foram usados tantos bits quanto possível, até obter a máxima capacidade do quadro para cada SF. Para todos os SF foram usados 64 bits de preâmbulo.

Tabela 4. Tamanho dos quadros para cada fator de espalhamento.

\begin{tabular}{lc}
\hline Fator de espalhamento & Tamanho do quadro (bits) \\
\hline SF7 & 904 \\
SF8 & 864 \\
SF9 & 832 \\
SF10 & 760 \\
SF11 & 736 \\
\hline
\end{tabular}

O controlador da unidade transmissora também envia o conteúdo das mensagens para sua interface USB e um sinal quando a mensagem não é respondida por um receptor, após um intervalo de tempo configurado. O controlador da unidade receptora, por sua vez, executa um código que recebe pacotes. A cada pacote recebido, são medidos a potência do sinal do enlace, a relação sinal-ruído (SNR) e também é obtida uma marca de tempo. O controlador da unidade receptora, então, envia o pacote recebido e os dados medidos para a interface USB. O controlador Arduino Yún da unidade receptora é conectado por sua interface USB (Universal Serial Bus) a um notebook, para que os pacotes e os dados sobre eles sejam armazenados. O controlador da unidade receptora tem um sistema operacional Linux embarcado, visado para implementar outras funcionalidades no protótipo.

\subsection{Cenário dos Experimentos}

O cenário utilizado para os experimentos e as medições para avaliação da tecnologia LoRa foi o campus da Ilha do Fundão da Universidade Federal do Rio de Janeiro (UFRJ). Foi estabelecido um ponto para a localização da unidade receptora e cinco pontos para a localização da unidade transmissora, de forma que, em diferentes momentos, a unidade transmissora estivesse a uma distância de $0 \mathrm{~m}, 500 \mathrm{~m}, 1000 \mathrm{~m}, 1500 \mathrm{~m}$ e $2000 \mathrm{~m}$ da unidade receptora, como mostrado na Figura 4(a). Buscou-se locais que possuíssem o mínimo de fontes de interferência entre si, com preferência a locais entre os quais houvesse linha de visão entre a unidade transmissora e a unidade receptora, na Figura 4(b).

A unidade receptora estava localizada no sétimo andar do bloco A do Centro de Tecnologia da UFRJ, destacado na Figura 4(a) como ponto em $0 \mathrm{~m}$. Para cada valor de distância, a unidade transmissora é localizada em um local diferente mostrado na Figura 4(a). Os experimentos são realizados no período do dia, com poucas nuvens, temperatura variando entre $32^{\circ} \mathrm{C}$ e $35^{\circ} \mathrm{C}$, umidade variando entre $65 \%$ e $75 \%$, com tráfego de veículos normal. Para cada distância entre a unidade transmissora e a unidade receptora, dez sequências de pacotes numerados de 0 a 50 são enviadas. 


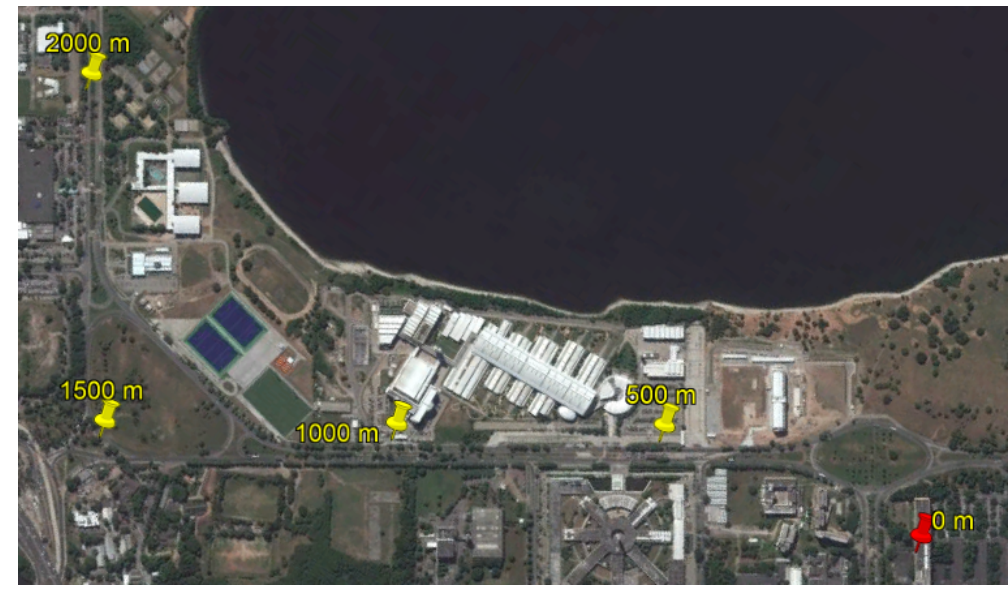

(a)

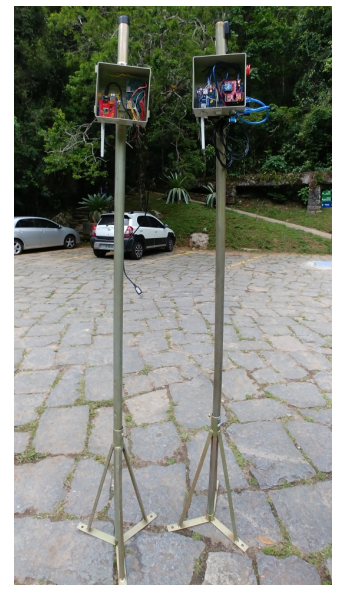

(b)

Figura 4. Cenário e equipamentos do protótipo desenvolvido. (a) Local dos experimentos. (b) Protótipos do experimento. À esquerda, unidade receptora. À direita, unidade transmissora.

\section{Resultados Experimentais}

Esta seção apresenta os resultados obtidos no envio de pacotes da unidade transmissora para a unidade receptora. São apresentados resultados para a taxa de perda, potência de sinal, SNR e vazão. Todos os resultados são apresentados com intervalo de confiança de $95 \%$, representado por barras de erro verticais nos gráficos.

\subsection{Medidas de Taxa de Perda de Pacotes}

Os resultados associados à taxa de perda do envio de pacotes entre as duas unidades são apresentados na Figura 5. Para isso, foi avaliada a porcentagem de pacotes perdidos a diferentes distâncias, para diferentes valores de SF. Enquanto o SF11 apresenta uma certa quantidade de perdas na distância de $0 \mathrm{~m}$ verifica-se pela barra de erro que os resultados variaram bastante, apontando para alguma fonte de interferência ocasional. Já o SF7 teve a maior perda entre todas as configurações, à maior distância, de $2000 \mathrm{~m}$. Porém, na distância de $1000 \mathrm{~m}$, observa-se uma taxa de perda de $25 \%$. Acredita-se que esta degradação do sinal representada em perda de pacotes para o SF7 está associada à vegetação presente no cenário no qual foi desenvolvido o experimento.

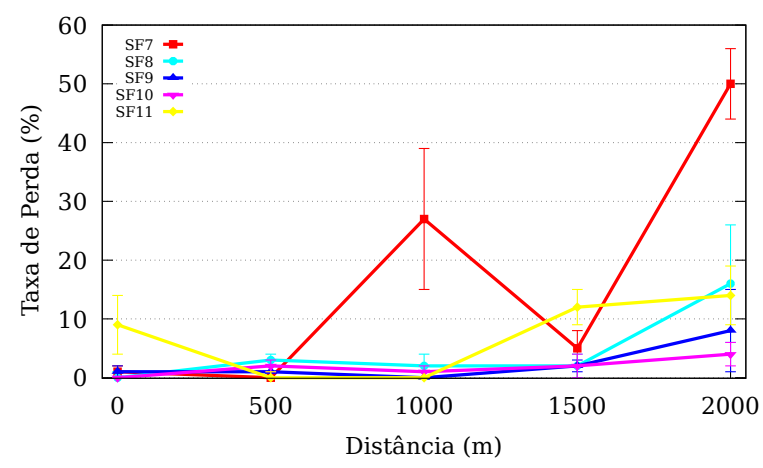

Figura 5. Taxa de perda medida nos experimentos com o protótipo produzido. 
A vegetação do local pode ter gerado obstáculos suficientes para impedir a entrega de uma significativa proporção dos pacotes. O ponto de $1000 \mathrm{~m}$ de distância é o único no qual há alguma vegetação entre a unidade transmissora e a unidade receptora. Para os outros fatores de espalhamento, o comportamento foi de acordo com esperado: a perda em maiores distâncias é reduzida conforme há um aumento no fator de espalhamento. Por exemplo, o fator SF7 apresenta uma perda de 50\% de pacotes em $2000 \mathrm{~m}$, enquanto o SF11 apresenta uma perda de $14 \%$.

\subsection{Medidas de Potência do Sinal}

A Figura 6 mostra o comportamento da potência do sinal no enlace. Inicialmente, a queda de potência é abrupta, diminuindo de $-40 \mathrm{dBm}$ para $-112 \mathrm{dBm}$ em $500 \mathrm{~m}$, e logo mantendo-se estável conforme há um aumento da distância, até os $-124 \mathrm{dBm}$. Com isso, a atenuação da potência do sinal do enlace diminui proporcionalmente com o aumento da distância, e, pelas características de potência de sinal do enlace com a tecnologia LoRa (sensibilidade de recepção de dados até $-130 \mathrm{dBm}$ ), é possível concluir que o enlace com o hardware implementado pode ter um maior alcance do que foi possível atingir nos experimentos. Também é possível observar que na distância de $1000 \mathrm{~m}$, o SF7 teve a menor potência do sinal, o que se associa a fatores ambientais de vegetação densa, apresentando uma queda de potência de aproximadamente $-4 \mathrm{dBm}$ em comparação com a potência do sinal para a seguinte distância $(1500 \mathrm{~m})$. Todos os fatores de espalhamento testados apresentam uma queda de potência no ponto de $1000 \mathrm{~m}$.

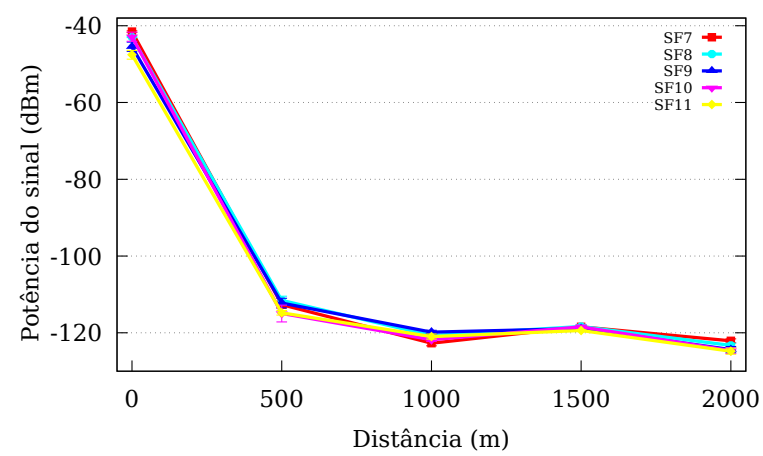

Figura 6. Potência do sinal para cada distância avaliada.

\subsection{Medidas da Relação Sinal-Ruído}

Outro dos resultados obtidos no experimento está associado à SNR. Sendo um indicador importante para representar a qualidade do sinal, a SNR permite predizer a quão boa pode ser a comunicação por um enlace. Assim, foram obtidos dados da SNR no experimento, apresentados na Figura 7. Pode-se detalhar que são obtidos valores positivos de SNR, o que indica que a potência do sinal é maior que a potência do ruído. Já os valores negativos indicam que a potência do sinal recebido é menor que a potência do ruído, porém, não o suficiente para afetar o enlace. Por outro lado, pode-se observar que a menor SNR apresenta-se no SF11, porém, também observa-se que independente da taxa de transmissão usada, a SNR descresce assim como aumenta a distância. 


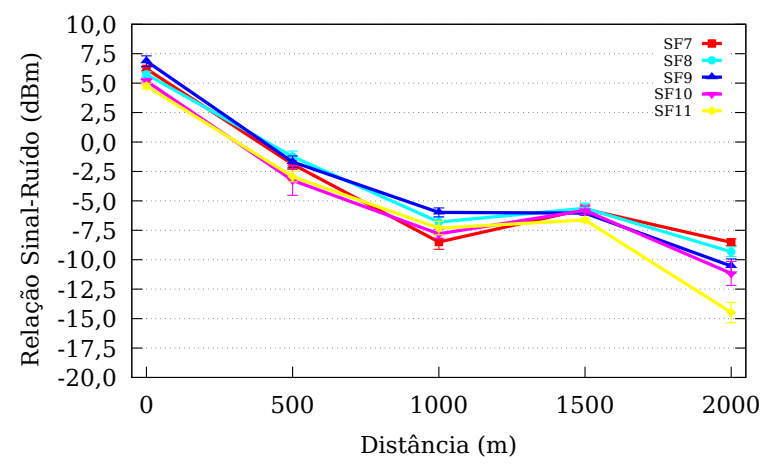

Figura 7. Relação Sinal-Ruído para o protótipo desenvolvido.

\subsection{Medidas de Vazão}

Foi avaliada a vazão do enlace baseado no tempo de chegada do primeiro pacote, e o tempo de chegada do último pacote para cada SF, o tamanho do quadro para cada SF, e cada distância. Como se pode observar na Figura 8, a máxima vazão foi obtida para o SF7, pois esse está configurado para transmitir uma maior quantidade de dados, porém, como já foi mostrado na avaliação da taxa de perda e a potência do sinal, o SF7 tem uma queda abrupta na vazão em $1000 \mathrm{~m}$. Os outros resultados concordam com o que tem sido descrito da tecnologia: em distâncias maiores, a taxa de transmissão de dados é menor, assim como o tempo de transmissão é maior, o que se pode ver representado na redução da taxa de transmissão para cada SF.

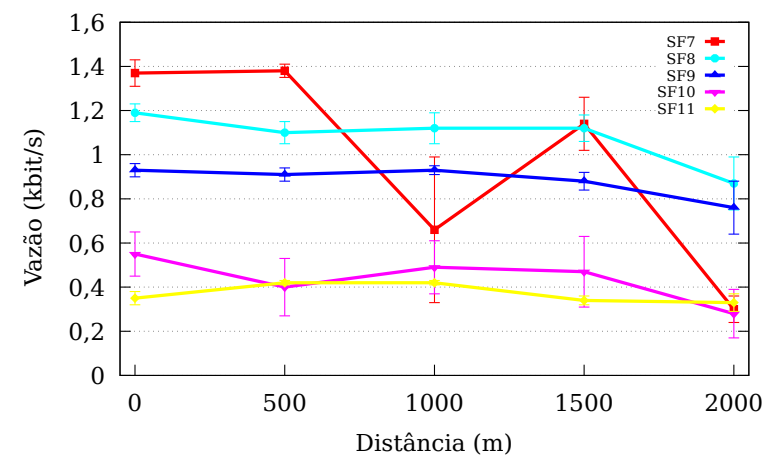

Figura 8. Vazão para cada distância segundo o fator de espalhamento LoRa.

\subsection{Comparação da Vazão Obtida com a Vazão Teórica}

A partir dos valores calculados para a taxa de transmissão teórica de cada fator de espalhamento, exibidos na Tabela 1, e também dos valores para a taxa de transmissão medidos com o protótipo, ilustrados na Figura 8, é possível comparar as taxas obtidas na prática com as taxas teóricas. A Figura 9 ilustra o valor percentual das taxas de transmissão obtidas na prática quando comparadas com as taxas de transmissão teóricas. Os resultados obtidos mostram uma porcentagem baixa de relação entre as taxas de transmissão teórica e prática. Isso está associado à implementação de retardos no protótipo implementado, mensagens de confirmação de chegada dos pacotes, que geram atraso na entrega dos pacotes avaliados. Porém, não impede dizer que o padrão de comunicação está dentro das taxas que é possível transmitir para a tecnologia. 


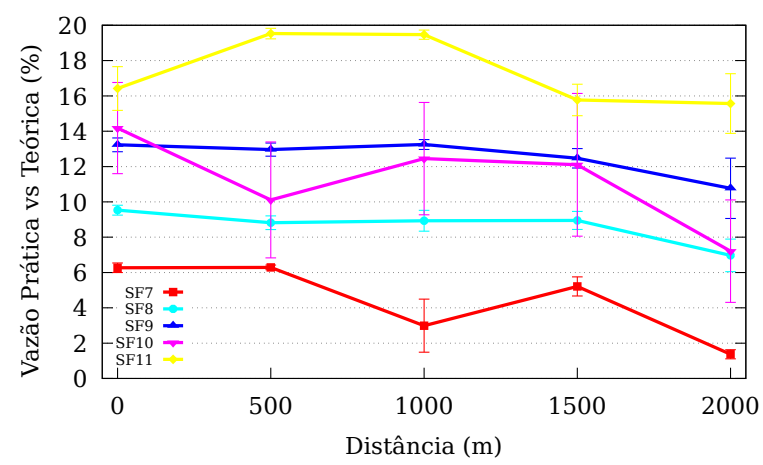

Figura 9. Comparação da vazão medida com a vazão teórica segundo o fator de espalhamento LoRa.

\section{Conclusão e Trabalhos Futuros}

No paradigma da Internet das Coisas (Internet of Things - IoT), objetos do cotidiano passam a ser capazes de atuar, sensoriar, processar e se comunicar. Esses objetos passam a ser chamados, então, de objetos inteligentes. As redes LPWAN possuem grande gama de aplicações, em especial sob o ponto de vista do paradigma IoT, pois os objetos inteligentes, em geral, são restritos em termos de energia.

A tecnologia LoRa é utilizada para implementar redes LoRaWAN e tem se tornado uma opção cada vez mais adotada comercialmente. Este trabalho conduziu uma caracterização da tecnologia LoRa para redes LPWAN. Essa tecnologia utiliza uma série de parâmetros para garantir a comunicação de longo alcance e baixa potência entre dispositivos, entre eles o fator de espalhamento (Spreading Factor - SF). O SF aumenta a sensibilidade do receptor, ao custo de menores taxas de transmissão. Foi construído um protótipo com dois nós capazes de trocar mensagens utilizando a tecnologia LoRa. A partir do envio e recepção de mensagens realizado pelo protótipo, são obtidas medidas de taxa de perda de pacotes, potência do sinal, relação sinal-ruído e de vazão. Essas medidas são obtidas variando o SF e a distância entre os nós do protótipo. As taxas de transmissão obtidas empiricamente são, então, comparadas com as taxas de transmissão teóricas, para cada SF utilizado. Segundo nossos resultados, o melhor desempenho com relação à taxa de transmissão teórica é o do maior fator de espalhamento testado, SF11. Isso se deve ao fato de, nessa configuração, há uma menor perda de pacotes. Com os resultados obtidos, observou-se a influência do SF no desempenho da tecnologia LoRa. A saber, não se tem um fator de espalhamento mais robusto que outro ou não, observa-se a degradação da comunicação quando as propriedades desse SF já não atingem a necessidade da comunicação. Como trabalhos futuros, pretende-se programar os nós do protótipo para que realizem retransmissões sempre com fatores de espalhamento mais altos. Assim, as retransmissões possuem uma chance maior de sucesso. Da mesma forma, pretende-se implementar um sistema de taxa adaptativo em arquiteturas de baixo custo, com o intuito de variar o SF segundo os níveis de potência do enlace, isto com o objetivo de aproveitar a propriedade de ortogonalidade e diversidade do SF na rede.

\section{Referências}

Atzori, L., Iera, A. e Morabito, G. (2010). The Internet of things: A survey. Computer Networks, 54(15):2787 - 2805. 
Baharudin, A. M. e Yan, W. (2016). Long-range wireless sensor networks for geo-location tracking: Design and evaluation. Em 2016 International Electronics Symposium (IES), p. $76-80$.

Bankov, D., Khorov, E. e Lyakhov, A. (2016). On the limits of LoRaWAN channel access. Em International Conference on Engineering and Telecommunication (EnT), p. 10-14.

Bor, M., Vidler, J. e Roedig, U. (2016). Lora for the internet of things. Em Proceedings of the 2016 International Conference on Embedded Wireless Systems and Networks, EWSN '16, p. 361-366, USA. Junction Publishing.

Centenaro, M., Vangelista, L., Zanella, A. e Zorzi, M. (2016). Long-range communications in unlicensed bands: the rising stars in the iot and smart city scenarios. IEEE Wireless Communications, 23(5):60-67.

Kim, D. H., Lim, J. Y. e Kim, J. D. (2016). Low-power, long-range, high-data transmission using Wi-Fi and LoRa. Em 2016 6th International Conference on IT Convergence and Security (ICITCS), p. 1-3.

LoRa-Alliance (2015a). Lora alliance wide area network for IoT. https://www.loraalliance.org/technology. Acessado em dezembro de 2017.

LoRa-Alliance (2015b). LoRaWAN ${ }^{\mathrm{TM}}$ specification. https://www.loraalliance.org/lorawan-for-developers. Acessado em dezembro de 2017.

Neumann, P., Montavont, J. e Noël, T. (2016). Indoor deployment of low-power wide area networks (LPWAN): A LoRaWAN case study. Em IEEE 12th International Conference on Wireless and Mobile Computing, Networking and Communications (WiMob), p. $1-8$.

Petäjäjärvi, J., Mikhaylov, K., Pettissalo, M., Janhunen, J. e Iinatti, J. (2017). Performance of a low-power wide-area network based on LoRa technology: Doppler robustness, scalability, and coverage. International Journal of Distributed Sensor Networks, 13(3):1-16.

Petäjäjärvi, J., Mikhaylov, K., Roivainen, A., Hanninen, T. e Pettissalo, M. (2015). On the coverage of LPWANs: range evaluation and channel attenuation model for LoRa technology. Em 14th International Conference on ITS Telecommunications (ITST), p. $55-59$.

Raza, U., Kulkarni, P. e Sooriyabandara, M. (2017). Low power wide area networks: An overview. IEEE Communications Surveys Tutorials, 19(2):855-873.

Schaffers, H., Komninos, N., Pallot, M., Trousse, B., Nilsson, M. e Oliveira, A. (2011). Smart Cities and the Future Internet: Towards Cooperation Frameworks for Open Innovation, p. 431-446. Springer Berlin Heidelberg, Berlin, Heidelberg.

Semtech Corporation (2013). LoRa modem designer's guide. Application Note AN1200.13.

Semtech Corporation (2015). Lora ${ }^{\mathrm{TM}}$ modulation basics. Application Note AN1200.

Toldov, V., Meijers, J., Igual-Pérez, R., Wolhuter, R., Mitton, N. e Clavier, L. (2016). Performance evaluation of LoRa radio solution for PREDNET wildlife animal tracking project. Em LPWAN 2016, Paris Roissy, France. 